\title{
Altered microstructure of brain white matter in females with anorexia nervosa: a diffusion tensor imaging study
}

This article was published in the following Dove Press journal:

Neuropsychiatric Disease and Treatment

Number of times this article has been viewed

\author{
Shao-Hua $\mathrm{Hu}^{1,2}$ \\ Hong Feng ${ }^{3,4}$ \\ Ting-Ting $X_{u^{3,5}}$ \\ Hao-Rong Zhang ${ }^{6}$ \\ Zhi-Yong Zhao ${ }^{6}$ \\ Jian-Bo Lai ${ }^{1,2}$ \\ Dong-Rong $\mathrm{Xu}^{7}$ \\ Yi Xul,2 \\ 'Department of Psychiatry, First \\ Affiliated Hospital, Zhejiang \\ University School of Medicine, \\ Hangzhou, ${ }^{2}$ The Key Laboratory \\ of Mental Disorder's Management \\ of Zhejiang Province, Hangzhou, \\ ${ }^{3}$ Faculty of Clinical Medicine, Zhejiang \\ University School of Medicine, \\ Hangzhou, ${ }^{4}$ Department of Psychiatry, \\ Shaoxing No Seven People's \\ Hospital, Shaoxing, ${ }^{5}$ Department of \\ Psychiatry, The Center of Mental \\ Health, Xiaoshan Hospital, Hangzhou, \\ ${ }^{6}$ Shanghai Key Laboratory of Magnetic \\ Resonance, Key Laboratory of Brain \\ Function Genomics, East China \\ Normal University, Shanghai, China; \\ ${ }^{7}$ Epidemiology Division and MRI \\ Unit, Department of Psychiatry, \\ Columbia University and New York \\ State Psychiatric Institute, New York, \\ NY, USA
}

Correspondence: Dong-Rong Xu Epidemiology Division and MRI Unit, Department of Psychiatry, Columbia University and New York State Psychiatric Institute, New York, NY 10032, USA

Email xud@nyspi.columbia.edu

YiXu

Department of Psychiatry, First Affiliated Hospital, Zhejiang University School of Medicine, Hangzhou 310003, Zhejiang, China

Email xuyizju@zju.edu.cn
Objective: Structural studies have reported anorexia nervosa (AN) patients with abnormal gray matter in several brain regions and dysfunction in some connected neural circuits. However, the role of white matter (WM) in AN patients has rarely been investigated. The present study aimed to assess alterations in WM microstructure of the entire brain in females with AN using a voxel-based method on diffusion tensor imaging (DTI) data.

Materials and methods: The study enrolled 8 female patients with AN and 14 age-matched females as controls $(\mathrm{CW})$. The DTI data was collected from each subject to calculate the fractional anisotropy (FA) maps of the whole brain by the DTI-Studio software. Subsequently, a 2 -sample $t$-test $(P<0.05$, corrected) was performed to detect the difference in FA maps of AN and CW group, and a Pearson's correlation analyzed the relationship between mean FA value of brain regions and body mass index (BMI).

Results: Compared with CW, AN patients revealed a significant decrease in FA maps in the left superior frontal gyrus, medial frontal gyrus, anterior cingulate cortex, middle frontal gyrus, inferior frontal gyrus, thalamus, and bilateral insula. Moreover, significantly positive correlations were established between the mean FA value of the left inferior frontal gyrus, insula as well as thalamus and BMI in AN patients.

Conclusions: Our findings supported the presence of WM abnormality in patients with AN. The significant differences of FA maps, in patients with AN, were associated with their aberrant BMI. The results further improved our understanding of the pathophysiological mechanisms underlying AN.

Keywords: anorexia nervosa, magnetic resonance imaging, diffusion tensor imaging, white matter

\section{Introduction}

Anorexia nervosa (AN) is a severe psychiatric eating disorder of unknown etiology related to self-driven food refusal and emaciation, altered body perception, and preoccupations with weight and shape. ${ }^{1}$ It is primarily characterized by disrupted eating patterns or weight-control behavior resulting in heightened anxiety and significantly increased damage to health, causing impaired physical and psychosocial functions. ${ }^{2-4}$ Because of its low therapeutic compliance and high expulsion rate, it has been a crucial factor of adolescent deaths in recent years. ${ }^{5}$

Recent studies demonstrated that the structural abnormality of the brain was a prerequisite for the development of AN. ${ }^{6}$ Several studies have shown alterations of gray matter volume (GMV), using voxel-wise analysis, in AN patients compared with the control group. For instance, Mühlau et al found a decreased global GMV and regional GM in 
the bilateral anterior cingulate cortex (ACC) in AN patients by $\sim 1 \%$ and $5 \%$, respectively, which remarkably correlated with the lowest body mass index (BMI). ${ }^{2}$ Boghi et al found a significant reduction in total white matter (WM) volume and local GM atrophy in cerebellum, hypothalamus, caudate nucleus, and frontal, parietal and temporal areas. In addition, an association between BMI and GM was also found in the hypothalamus. ${ }^{7}$ Fonville et al detected smaller global GMV and regional decrease in the cerebellum, temporal, frontal, and occipital lobes in adult participants with $\mathrm{AN}$, and a relation between GMV loss and duration of illness in the cerebellum and mesencephalon. ${ }^{8}$ Moreover, some of these volume depletions may return to normal in recovered patients, ${ }^{9}$ while other studies have shown their persistence..$^{2,10,11}$

Structural neuroimaging studies of AN primarily concentrate on alterations of gray matter. To date, study focused on the abnormality of WM is rare. Brain WM axons connect between cortical and subcortical brain structures and thus have a critical impact on cognitive and emotional processing. ${ }^{12}$ Diffusion tensor imaging (DTI), an advanced magnetic resonance (MR) technique, can reveal the microstructural integrity of WM pathways. ${ }^{13}$ One of the most important DTI metrics, fractional anisotropy (FA) is capable of measuring water diffusion along the WM tracts. It has been previously applied in other psychiatric disorders to investigate the change of WM microstructure. ${ }^{14}$ Lower FA inversely affects the WM integrity because of either fragile axonal coherence, density or myelination. ${ }^{15,16}$ A recent DTI study suggested that WM integrity of AN patients was destroyed in limbic and association pathways, including fimbria-fornix, fronto-occipital, and posterior WM cingulum. The fimbriafornix WM integrity can also predict the harm avoidance. ${ }^{12}$ Another tract-based study found that AN patients showed significant FA decrease in the parietal part of the left superior longitudinal fasciculus, with increased mean diffusion (MD) and radial diffusion (RD) but unaltered axial diffusion (AD). Patients with AN also revealed remarkably increased MD in the fornix, accompanied by low FA, and high RD and AD. ${ }^{17}$ However, these studies mainly focused on the altered WM integrity in several fiber tracts related to $\mathrm{AN}$, but studies investigating WM microstructure of the whole brain using voxel-based method are scarce.

Although a recent voxel-based DTI study on AN has been reported, the outcomes presented only reduced FA value in the bilateral posterior thalamic radiation, while the correlation analysis between decreased FA value and BMI of AN patients was not explored. ${ }^{6}$ Previous studies have demonstrated that the common cognitive dysfunctions, such as executive functions, visuospatial processes, self-image perception, emotional regulation, and reward processing, were found in AN patients. ${ }^{18}$ Therefore, to explore the mechanism underlying the etiology of AN, the voxel-based analysis of the entire brain on DTI data was performed between AN and controls in the present study. We hypothesized that the altered FA would be found in widespread regions, such as prefrontal cortex (PFC), parietal lobe, and subcortical regions, that are closely associated with the impaired functions in AN patients. Concurrently, significant correlations would be exhibited between altered FA and BMI in AN patients.

\section{Materials and methods \\ Participants}

Eight right-handed female patients with AN (average age $17.6 \pm 2.2$ years, average height $162 \pm 3.0 \mathrm{~cm}$ ), fulfilling Diagnostic and Statistical Manual of Mental Disorders fourth edition text revision criteria for the restricted subtype of $\mathrm{AN},{ }^{19}$ from the Department of Psychiatry, First Affiliated Hospital of Zhejiang University School of Medicine, China, were consecutively recruited. In addition, 14 controls (females, average age $19.1 \pm 3.1$ years, average height $161 \pm 3.9 \mathrm{~cm}$ ) matched in age and height $(P=0.239, P=0.600$, respectively, 2-sample 2-tailed Student's $t$-test), gender, and dexterity, were recruited via proximal advertisements. Comorbid psychiatric disorders, neurologic conditions, and abuse of any substance except nicotine comprised the exclusion criteria. Before scanning, all patients were required to have had at least 1 week of supervised meals and hydration during their regular hospital admission. This minimized the alleged biases caused by dehydration in brain measurements. ${ }^{20}$ All controls were screened using the General Health Questionnaire and a clinical semi-structured interview to exclude any psychiatric or other medical condition. ${ }^{21} \mathrm{We}$ also ensured that the controls had a BMI within the healthy range (average BMI, $20.1 \pm 1.7 \mathrm{~kg}^{*} \mathrm{~m}^{-2}$ ) and that they did not present unhealthy eating behaviors (eg, constant dieting) or sub-threshold symptoms of any eating disorder. The demographic characteristics of each patient are displayed in Table 1. This study was approved by Institutional Ethics Committee of the First Affiliated Hospital of Zhejiang University School of Medicine. Informed consent was obtained from all participants or legal guardians for those under the legal age of consent.

\section{Image acquisition and processing}

Participants were scanned using a 3T MR imaging system (GE sign HDXM) with an 8-channel phased array head coil. Cushions restricted head movements, and the participants 
Table I Demographic characteristics and clinical assessment of AN patients

\begin{tabular}{|c|c|c|c|c|c|c|c|}
\hline ID & Age (years) & Gender & Handedness & Duration (month) & Height (cm) & Weight (kg) & BMI $\left(\mathrm{kg} / \mathrm{m}^{2}\right)$ \\
\hline 01 & 16 & $\mathrm{~F}$ & $\mathrm{R}$ & 6 & 162 & $32.5 / 50^{\mathrm{a}}$ & 12.7 \\
\hline 02 & 18 & $\mathrm{~F}$ & $\mathrm{R}$ & 12 & 164 & $40 / 54$ & 14.9 \\
\hline 03 & 17 & $\mathrm{~F}$ & $\mathrm{R}$ & 12 & 160 & $42.5 / 48$ & 16.6 \\
\hline 04 & 22 & $\mathrm{~F}$ & $\mathrm{R}$ & 6 & 168 & $37 / 51$ & 13.1 \\
\hline 05 & 15 & $\mathrm{~F}$ & $\mathrm{R}$ & 6 & 158.5 & $33.5 / 42$ & 13.3 \\
\hline 06 & 16 & $\mathrm{~F}$ & $\mathrm{R}$ & 6 & 163 & $38 / 44$ & 14.4 \\
\hline 07 & 19 & $\mathrm{~F}$ & $\mathrm{R}$ & 24 & 160 & $40 / 44$ & 15.6 \\
\hline 08 & 18 & $\mathrm{~F}$ & $\mathrm{R}$ & 12 & 161 & $36 / 51$ & 13.9 \\
\hline
\end{tabular}

Note: ${ }^{a}$ Represents the standard weight for each patient.

Abbreviations: AN, anorexia nervosa; BMI, body mass index; $F$, female; $R$, right-handed.

wore earplugs. DTI data was obtained using a single-shot echo-planar imaging sequence in 44 axial planes with 25 nonlinear diffusion sensitization gradients $\left(b=1,000 \mathrm{~s} / \mathrm{mm}^{2}\right)$, and a reference image without diffusion weighting (b0-image). Imaging parameters were: repetition time/echo time (TR/TE), 12,000/89 ms; slice thickness, $3 \mathrm{~mm}$ (no slice gap); number of excitations, 2; matrix, 128 $\times 128$; field of view (FOV), $240 \times 240 \mathrm{~mm}^{2}$; voxel size, $1 \times 1 \times 3 \mathrm{~mm}^{3}$. DTI was performed using axial sections parallel to the anterior-posterior commissural line to cover the entire brain. As an anatomical reference, high-resolution structural images were acquired using a magnetized rapid gradient-echo (MPRAGE) 3-dimensional T1-weighted sequence: TR/TE, 7.1/2.9 ms; 146 slices with thickness $1 \mathrm{~mm}$; flip angle, $8^{\circ}$; matrix, 256×256; FOV, $240 \times 240 \mathrm{~mm}^{2}$; voxel size, $1 \times 1 \times 1 \mathrm{~mm}^{3}$. DTI data processing and analysis were performed using DTI-Studio program SPM8 (Johns Hopkins Medical Institute, Baltimore, ML, USA), and software tools developed in-house. Subsequently, the echo planar distortions induced by eddy-current were corrected using an affine transformation algorithm, following which the parametric maps of FA were created to perform the voxel-wise analysis and region of interest (ROI) analysis.

\section{Voxel-wise analysis of FA maps between $\mathrm{AN}$ and $\mathrm{CW}$}

The voxel-wise of FA maps was conducted by using statistical parametric mapping (SPM8). First, b0 images of each CW were normalized to Montreal Neurological Institute space by an echo-planar imaging template, and then averaged. Next, the averaged images were spatially leveled using an anisotropic Gaussian filter with a full width at half maximum of $6 \mathrm{~mm}$. Thus, we obtained a new template of $\mathrm{b} 0$ image in the Montreal Neurological Institute space. Subsequently, individual b0 images of all patients and $\mathrm{CW}$ were registered to the new template (the normalization used the nonlinear registration method in SPM, including affine transformation and nonlinear transformation) and corresponding transformation matrices were also obtained. FA maps of each participant were then normalized to Montreal Neurological Institute space by using their corresponding transformation matrix and leveled by an anisotropic Gaussian filter with a full width at half maximum of $6 \mathrm{~mm}$. Finally, a two-sample Student's $t$-test was performed on the leveled FA of patients and $\mathrm{CW}$. The threshold was set at a combined cut-off value of $P<0.05$ (false discovery rate corrected) and a minimal cluster size of 50 voxels.

\section{ROI analysis of FA maps}

According to the significant differences in FA maps detected by voxel-wise analysis, we identified and analyzed the ROIs to quantify further the degree of secondary degeneration in patients compared with CW. First, we made binary masks by using the clusters with significant group differences of FA maps between groups. Then, the respective binary masks were applied to the normalized FA maps of all the participants. Finally, a value for each ROI was obtained by averaging all voxel values within each mask. Furthermore, correlation analysis was carried out between BMI and each ROI value across all patients and a $P$-value $<0.05$ was considered to be statistically significant.

\section{Clinical assessment}

The BMI is one of the most important markers of $\mathrm{AN}$, and its relevance has also been recognized from a general health perspective. A low BMI at the beginning of treatment has been identified as pivotal risk factors for poor prognosis in AN patients. BMI in the present study is used to evaluate the illness severity of AN, which is calculated as the body mass divided by the square of the height in each subject.

\section{Results \\ Voxel-wise analysis of FA maps}

Compared with $\mathrm{CW}$, the significantly decreased FA was found in the left superior frontal gyrus (SFG), medial frontal 


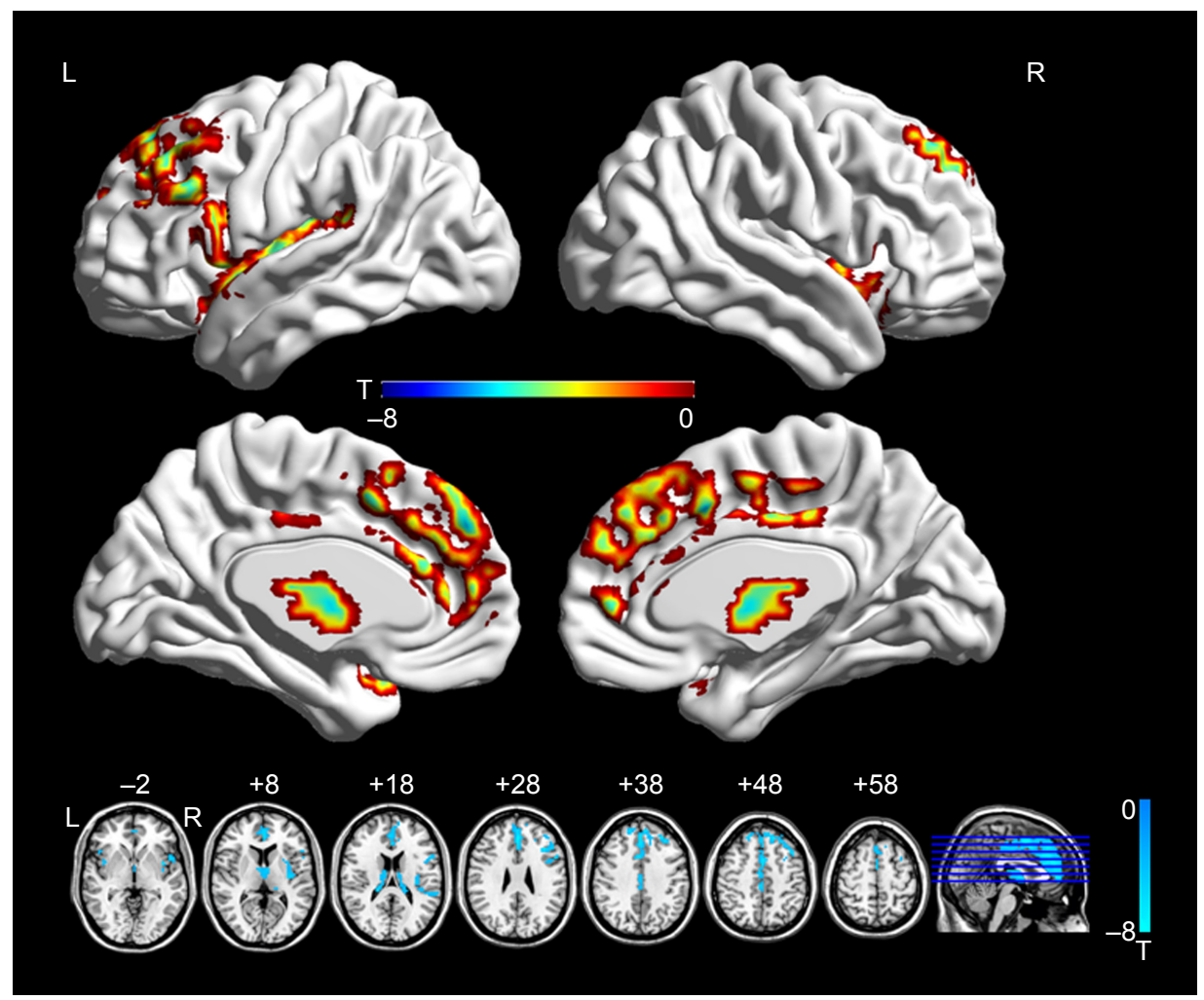

Figure I Brain regions showing decreased FA in the AN group relative to healthy controls.

Note: The color bars indicate the Student's $t$-test value and the statistical at $P<0.05$ (false discovery rate-corrected).

Abbreviations: AN, anorexia nervosa; FA, fractional anisotropy; L, left; R, right.

gyrus (medFG), ACC, middle frontal gyrus (MFG), inferior frontal gyrus (IFG), thalamus, and bilateral insula in AN groups (Figure 1; Table 2).

\section{ROI analysis of FA maps}

AN group showed reduced FA in the left $\mathrm{SFG}(P<0.01)$, medFG $(P<0.01)$, ACC $(P<0.01)$, MFG $(P<0.01)$, IFG $(P<0.01)$, thalamus $(P<0.01)$, and bilateral insula $(P<0.01)$ (Figure 2). More importantly, we found significantly positive correlations between the mean FA value of the

Table 2 Regions showing decreased FA in AN group

\begin{tabular}{|c|c|c|c|c|c|c|}
\hline \multirow[t]{2}{*}{ Regions } & \multirow[t]{2}{*}{ BA } & \multicolumn{3}{|c|}{$\begin{array}{l}\text { MNI } \\
\text { coordinates }\end{array}$} & \multirow[t]{2}{*}{ Cluster } & \multirow[t]{2}{*}{$\mathbf{T}$} \\
\hline & & $\mathbf{x}$ & $\mathbf{Y}$ & $\mathbf{Z}$ & & \\
\hline Left superior frontal gyrus & 9 & 0 & 3 & 51 & 193 & -7.50 \\
\hline Left medial frontal gyrus & 8 & 0 & 42 & 24 & 184 & -7.50 \\
\hline Left anterior cingulate cortex & 32 & & & & 118 & -4.83 \\
\hline Left middle frontal gyrus & $9 / 46$ & -36 & 24 & 27 & 57 & -6.67 \\
\hline Left inferior frontal gyrus & $44 / 45$ & -51 & 12 & 27 & 92 & -5.66 \\
\hline Left thalamus & - & -3 & -3 & 6 & 112 & -5.20 \\
\hline Left insula & 13 & -36 & 12 & -18 & 105 & -6.02 \\
\hline Right insula & 13 & 42 & 9 & -9 & 52 & -6.09 \\
\hline
\end{tabular}

Abbreviations: AN, anorexia nervosa; BA, Broca's area; FA, fractional anisotropy; MNI, Montreal Neurological Institute. left IFG $(r=0.916 ; P=0.001)$, insula $(r=0.898 ; P=0.002)$ as well as thalamus $(r=0.815 ; P=0.014)$ and $\mathrm{BMI}$ of $\mathrm{AN}$ patients (Figure 3).

\section{Reproducibility}

The sample size was relatively small in the present study. The reproducibility and robustness of these findings on FA were validated by a leave-one-out validation. Specifically, 1 AN patient was left out of the sample, and the group comparisons were performed based on the permutated samples (ie, $8 \mathrm{AN}$ vs $14 \mathrm{CW}$ ). This led to a total of 82 -sample $t$-test images; for each voxel, the number of tests where this voxel exhibited significant differences between groups was calculated as the reproducibility of FA differences between AN patients and CW. Figure 4 indicates the highly reproducible altered FA patterns across these tests as well as the original test reported.

\section{Discussion}

The findings of the present study supported our hypothesis that AN patients exhibit widespread changes of FA values in the regions related to AN compared with CW. Specifically, we found reduced FA values in the left SFG, medFG, ACC, 


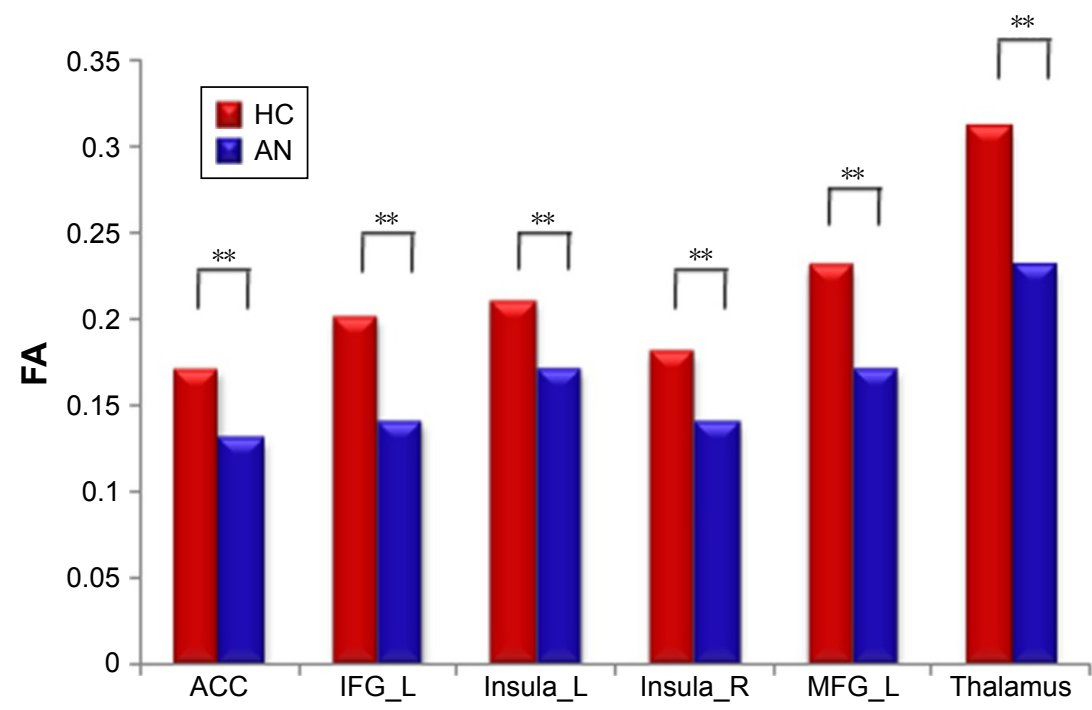

Figure $\mathbf{2}$ The mean FA of region of interests with differences between groups were displayed.

Note: $* * P<0.01$.

Abbreviations: ACC, anterior cingulate cortex; AN, anorexia nervosa; FA, fractional anisotropy; HC, healthy controls; IFG, inferior frontal gyrus; L, left; MFG, middle frontal gyrus; R, right.

MFG, IFG, thalamus, and bilateral insula in the patients with AN. Furthermore, significantly positive correlations were found between the mean FA value of the left IFG, insula as well as thalamus and BMI in AN patients, demonstrating that the altered FA value may reflect the degeneration of WM integrity in the particular regions of AN patients, which can be associated with the underlying AN mechanism.

$\mathrm{PFC}$, involved in regulating emotion and motivation, is a hub of the cognitive control network. ${ }^{22}$ On the other hand, the left anterior and posterior insula activation is associated
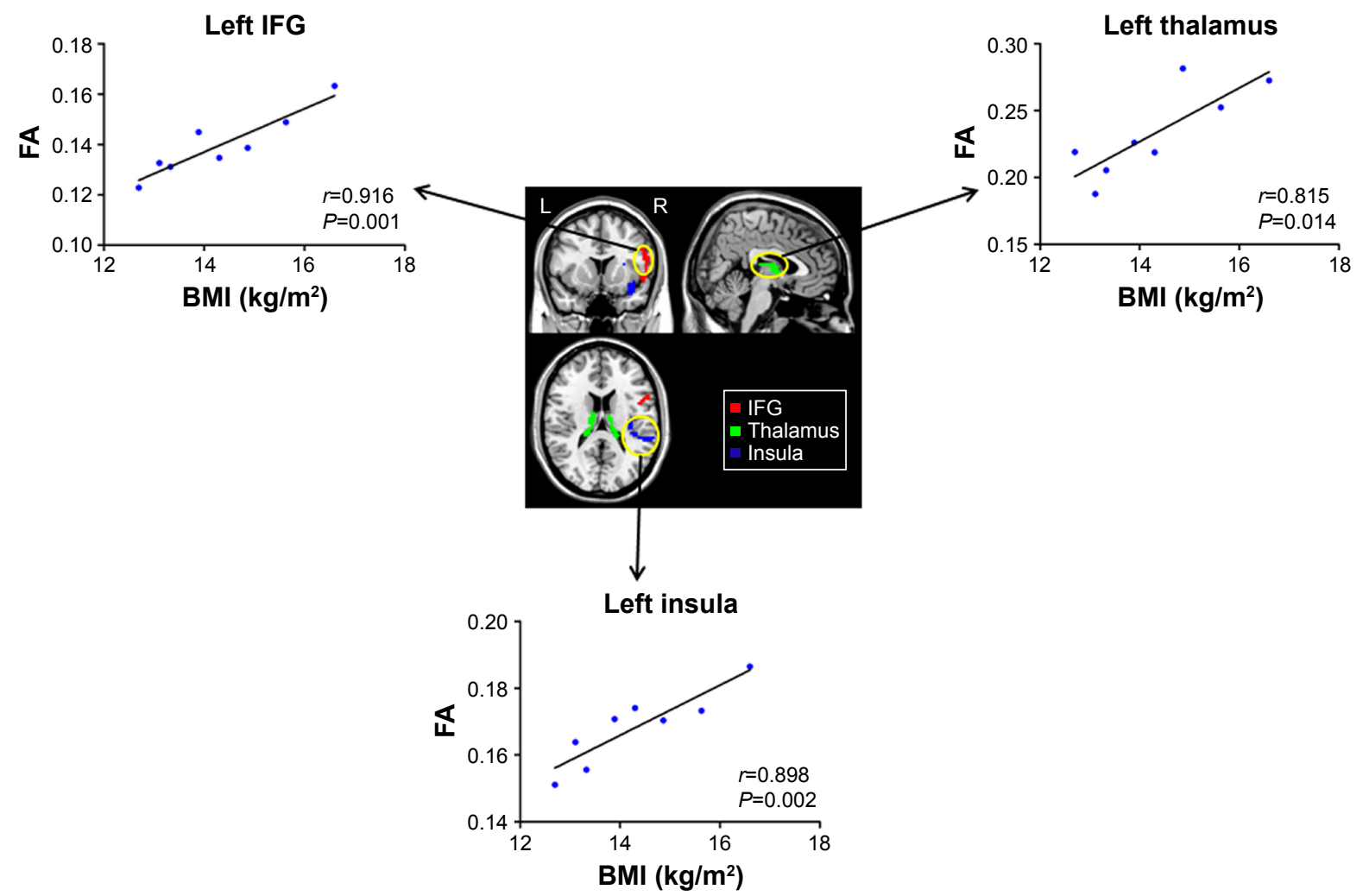

Figure 3 The clusters (yellow circles) were associated with significant differences between the AN and CW groups, and differences within which the FA index significantly correlated with BMI.

Abbreviations: AN, anorexia nervosa; BMI, body mass index; CW, control women; FA, fractional anisotropy; IFG, inferior frontal gyrus; L, left; R, right. 


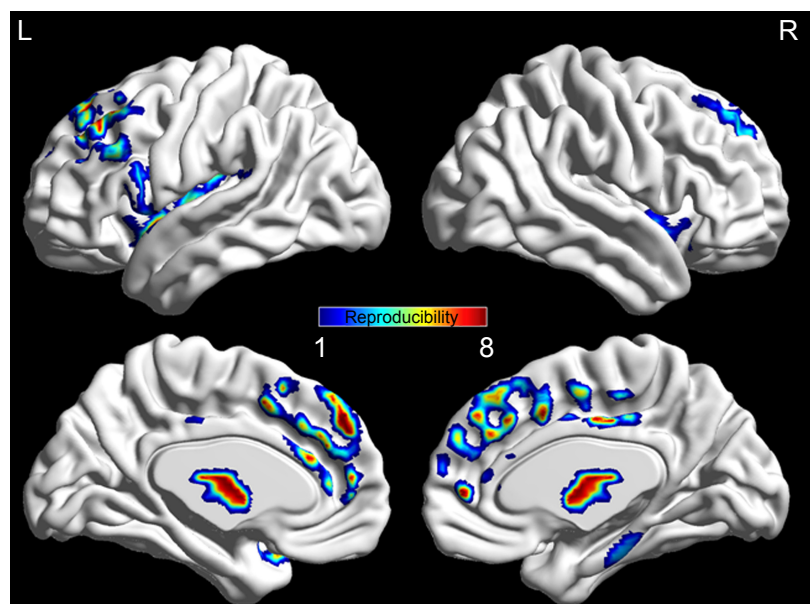

Figure 4 The reproducible patterns of FA variations in AN group. Abbreviations: AN, anorexia nervosa; FA, fractional anisotropy; L, left; R, right.

with gastric distension, and thus, is mediated by $\mathrm{BMI} ;{ }^{23}$ right anterior insula is connected with self-recognition, the "abstract representation of oneself," 24 and interoceptive awareness, ${ }^{25}$ and hence, a fixed perception of being fat while severely underweight presenting in AN patients. ${ }^{26}$ The altered signal was discovered in several structural and functional studies in patients with AN. In GM studies, reduced GMV was consistently observed in the prefrontal lobe and insula of AN patients compared with the control group. ${ }^{27-29}$ The taskbased functional magnetic resonance imaging study showed increased activations in the PFC, insula, and amygdala of AN patients, using a distorted fat body image of the patients as a visual task. These regions were composed of a network related to the affective component. ${ }^{30-32}$ In a study with AN and bulimia nervosa, the patterns of connectivity between the insula and frontal brain regions distinguished the groups during a visual food-cue task. ${ }^{33}$ A similar study of AN indicated reduced resting-state connectivity in cognitive control contributing area in the frontal cortex. ${ }^{34}$ On the study of WM, FA values in adolescents with AN were lower in the fornix, posterior frontal, and parietal areas, but higher in anterior frontal, orbitofrontal, and temporal lobes. ${ }^{35}$ Another study manifested positive correlations between BMI and FA, suggesting that weight loss could be related to the changes in FA. ${ }^{36}$ In agreement with previous studies, decreased FA values in AN patients were revealed in the PFC and insula in the present study, which positively correlated with BMI. This may indicate that the altered WM integrity in PFC and insula is associated with dysfunctional emotions and cognition control in AN patients.

The primary function of the thalamus is to filter and relay sensory information from the spinothalamic tract to other brain regions. ${ }^{37}$ Using graph theory method, a recent network analysis in patients with acute $\mathrm{AN}$, found locally decreased strength and degree, while increased path length in the middle/posterior insula and the thalamus. Reduced network efficiency in the thalamus and the middle/posterior insula may reflect the altered filtering and calibration of nociceptive, thermal, and other interoceptive signals that may contribute to abnormal representations of body size as well as hunger. ${ }^{37}$ The posterior thalamic radiation, involved in the processing of the body image revealed decreased $F A$ values in AN patients. ${ }^{6}$ Voxel-wise analysis also found reduced GMV in the extrastriate body area (EBA) in females suffering from acute AN. ${ }^{38}$ The parietal cortex region containing the EBA showed alteration of the diffusivity via the apparent diffusion coefficient-analysis. ${ }^{6}$ The left mediodorsal thalamic nucleus revealed reduced FA value, which is connected to the ACC, the PFC, and the ventral striatum. ${ }^{6}$ All of these regions have been implicated in the pathology of $\mathrm{AN}$, contributing to the impairments in cognitive domains, especially set-shifting ability, executive control and habit learning, and in reward processing. Decreased FA of the left thalamus in $\mathrm{AN}$ patients in the present study may suggest the degeneration of WM integrity in this region. Moreover, the significant positive correlation between mean FA in this region and BMI may indicate the structural basis of functional impairments of emotion and cognition induced by $\mathrm{AN}$ in the patients.

ACC involvement in the manifestation of psychiatric symptoms is well established, ${ }^{39,40}$ which appears to be a recent brain adaptation that is linked to cognitive and emotional processes unique to humans. ${ }^{41}$ Findings from functional neuroimaging studies as well as positron emission tomography highlight the role of ACC in AN patients. ${ }^{42-43}$ Recently, these outcomes were supported by several structural studies using voxel-wise analysis. For example, patients recovered from AN showed a reduced GMV in the anterior cingulum. ${ }^{2}$ A recent study using voxel-based morphometry in anorectic patients illustrated volume depletion in right anterior insular cortex, bilateral parahippocampal gyrus, left fusiform gyrus, left cerebellum, and right posterior cingulate while an increased volume of the right dorsolateral PFC was observed. ${ }^{28}$ Additionally, ACC plays a major role in the reward mechanism, ${ }^{44,45}$ which could be associated with setshifting deficit among AN patients. ${ }^{46}$ The reduced FA value of ACC found in AN patients in the present study indicated that the damage of WM in the ACC may provide the structural mechanism underlying the dysfunction of emotions and reward processes. 


\section{Limitations and future directions}

The present study uncovered abnormal FA in specific AN-related regions in female patients with $\mathrm{AN}$ using the voxel-wise analysis method on DTI data. Nevertheless, some limitations remain. First, the sample size was relatively small, and thus, further studies with larger sample size are indispensable. Second, body image distortion and other disorders with specific behavioral or cognitive phenomena and attitudes were not assessed; thus, we cannot evaluate whether there is a direct association between the observed microstructural abnormalities related to AN and the perceived body image distortion in the patients.

\section{Conclusion}

In this study, we have demonstrated that WM microstructural alterations are present in the patients with AN. By a voxelbased approach on DTI data, we identified regions with prominent FA changes and significant correlations with BMI. This is closely associated with current hypotheses about the pathology of AN.

\section{Acknowledgments}

This work was supported by the grants of the Medicine and Health Program of Zhejiang Province (No 2014ZDA008), the National Natural Science Foundation of China (81671357, 81471734), the National Key Basic Research Program (2016YFC1307100, 2017YFC1308502), the Public Welfare Project of Science Technology Department of Zhejiang Province (2015C33133), National Clinical Research Center for Mental Health Disorders (2015BAI13B02), and the Key Research Project of Zhejiang Province (2015C03040). We acknowledge Dr Pornkanok Prukpitikul for polishing the language of this paper.

\section{Disclosure}

The authors report no conflicts of interest in this work.

\section{References}

1. Nietzel M, Wakefield J. American psychiatric association diagnostic and statistical manual of mental disorders. Contemp Psychol. 1996;41: 642-651.

2. Mühlau M, Gaser C, Ilg R, et al. Gray matter decrease of the anterior cingulate cortex in anorexia nervosa. Am J Psychiatry. 2007;164(12):1850-1857.

3. Bulik CM, Sullivan PF, Fear JL, Pickering A. Outcome of anorexia nervosa: eating attitudes, personality, and parental bonding. Int J Eat Disord. 2000;28(2):139-147.

4. Klump KL, Strober M, Bulik CM, et al. Personality characteristics of women before and after recovery from an eating disorder. Psychol Med. 2004;34(8):1407-1418.

5. Papadopoulos FC, Ekbom A, Brandt L, Ekselius L. Excess mortality, causes of death and prognostic factors in anorexia nervosa. Br JPsychiatry. 2009;194(1):10-17.
6. Frieling H, Fischer J, Wilhelm J, et al. Microstructural abnormalities of the posterior thalamic radiation and the mediodorsal thalamic nuclei in females with anorexia nervosa-a voxel based diffusion tensor imaging (DTI) study. J Psychiatr Res. 2012;46(9):1237-1242.

7. Boghi A, Sterpone S, Sales S, et al. In vivo evidence of global and focal brain alterations in anorexia nervosa. Psychiatry Res. 2011;192(3): 154-159.

8. Fonville L, Giampietro V, Williams S, Simmons A, Tchanturia K. Alterations in brain structure in adults with anorexia nervosa and the impact of illness duration. Psychol Med. 2014;44(9):1965-1975.

9. Van den Eynde F, Suda M, Broadbent H, et al. Structural magnetic resonance imaging in eating disorders: a systematic review of voxel-based morphometry studies. Eur Eat Disord Rev. 2012;20(2):94-105.

10. Frank GK, Shott ME, Hagman JO, Mittal VA. Alterations in brain structures related to taste reward circuitry in ill and recovered anorexia nervosa and in bulimia nervosa. Am J Psychiatry. 2013;170(10): $1152-1160$.

11. Joos A, Hartmann A, Glauche V, et al. Grey matter deficit in longterm recovered anorexia nervosa patients. Eur Eat Disord Rev. 2011 19(1):59-63.

12. Kazlouski D, Rollin MD, Tregellas J, et al. Altered fimbria-fornix white matter integrity in anorexia nervosa predicts harm avoidance. Psychiatry Res. 2011;192(2):109-116.

13. Filler A. Magnetic resonance neurography and diffusion tensor imaging: origins, history, and clinical impact of the first 50,000 cases with an assessment of efficacy and utility in a prospective 5000-patient study group. Neurosurgery. 2009;65(4 Suppl):A29-A43.

14. White T, Nelson M, Lim KO. Diffusion tensor imaging in psychiatric disorders. Top Magn Reson Imaging. 2008;19(2):97-109.

15. Le Bihan D. Looking into the functional architecture of the brain with diffusion MRI. Nat Rev Neurosci. 2003;4(6):469-480.

16. Cohen MX, Schoene-Bake JC, Elger CE, Weber B. Connectivity-based segregation of the human striatum predicts personality characteristics Nat Neurosci. 2009;12(1):32-34.

17. Via E, Zalesky A, Sánchez I, et al. Disruption of brain white matter microstructure in women with anorexia nervosa. J Psychiatry Neurosci. 2014;39(6):367-375.

18. Kaye WH, Fudge JL, Paulus M. New insights into symptoms and neurocircuit function of anorexia nervosa. Nat Rev Neurosci. 2009;10(8) 573-584.

19. First MB, Spitzer RL, Gibbon M, Williams JB. Structured Clinical Interview for DSM-IV Axis I Disorders, Clinician Version. Washington, DC: American Psychiatric Association; 1997.

20. Streitbürger DP, Möller HE, Tittgemeyer M, Hund-Georgiadis M, Schroeter ML, Mueller K. Investigating structural brain changes of dehydration using voxel-based morphometry. PLoS One. 2012;7(8):e44195.

21. Goldberg DP. Manual of the General Health Questionnaire. England: NFER Publishing; 1978

22. Pessoa L. How do emotion and motivation direct executive control? Trends Cogn Sci. 2009;13(4):160-166.

23. Tomasi D, Wang GJ, Wang R, et al. Association of body mass and brain activation during gastric distention: implications for obesity. PLoS One 2009;4(8):e6847.

24. Devue C, Collette F, Balteau E, et al. Here I am: the cortical correlates of visual self-recognition. Brain Res. 2007;1143:169-182.

25. Critchley HD, Wiens S, Rotshtein P, Öhman A, Dolan RJ. Neural systems supporting interoceptive awareness. Nat Neurosci. 2004;7: 189-195.

26. Konstantakopoulos G, Varsou E, Dikeos D, et al. Delusionality of body image beliefs in eating disorders. Psychiatry Res. 2012;200(2-3): $482-488$.

27. Friederich HC, Walther S, Bendszus M, et al. Grey matter abnormalities within cortico-limbic-striatal circuits in acute and weight-restored anorexia nervosa patients. Neuroimage. 2012;59(2):1106-1113.

28. Brooks SJ, Barker GJ, O'Daly OG, et al. Restraint of appetite and reduced regional brain volumes in anorexia nervosa: a voxel-based morphometric study. BMC Psychiatry. 2011;11:179. 
29. Frank GK. Advances from neuroimaging studies in eating disorders. CNS Spectr. 2015;20(4):391-400.

30. Miyake Y, Okamoto Y, Onoda K, et al. Brain activation during the perception of distorted body images in eating disorders. Psychiatry Res. 2010;181(3):183-192.

31. Seeger G, Braus DF, Ruf M, Goldberger U, Schmidt MH. Body image distortion reveals amygdala activation in patients with anorexia nervosa-a functional magnetic resonance imaging study. Neurosci Lett. 2002;326(1):25-28.

32. Wagner A, Ruf M, Braus DF, Schmidt MH. Neuronal activity changes and body image distortion in anorexia nervosa. Neuroreport. 2003;14(17):2193-2197.

33. Kim KR, Ku J, Lee JH, Lee H, Jung YC. Functional and effective connectivity of anterior insula in anorexia nervosa and bulimia nervosa. Neurosci Lett. 2012;521(2):152-157.

34. Kullmann S, Giel KE, Teufel M, Thiel A, Zipfel S, Preissl H. Aberrant network integrity of the inferior frontal cortex in women with anorexia nervosa. Neuroimage Clin. 2014;4:615-622.

35. Frank GK, Shott ME, Hagman JO, Yang TT. Localized brain volume and white matter integrity alterations in adolescent anorexia nervosa. J Am Acad Child Adolesc Psychiatry. 2013;52(10):1066.e5-1075.e5.

36. Nagahara Y, Nakamae T, Nishizawa S, et al. A tract-based spatial statistics study in anorexia nervosa: Abnormality in the fornix and the cerebellum. Prog Neuropsychopharmacol Biol Psychiatry. 2014;51:72-77.

37. Geisler D, Borchardt V, Lord AR, et al. Abnormal functional global and local brain connectivity in female patients with anorexia nervosa. J Psychiatry Neurosci. 2016;41(1):6-15.
38. Suchan B, Busch M, Schulte D, Grönermeyer D, Herpertz S, Vocks S. Reduction of gray matter density in the extrastriate body area in women with anorexia nervosa. Behav Brain Res. 2010;206(1):63-67.

39. Yücel M, Wood SJ, Fornito A, Riffkin J, Velakoulis D, Pantelis C. Anterior cingulate dysfunction: implications for psychiatric disorders? J Psychiatry Neurosci. 2003;28(5):350-354.

40. Paus T. Primate anterior cingulate cortex: where motor control, drive and cognition interface. Nat Rev Neurosci. 2001;2(6):417-424.

41. McCormick LM, Keel PK, Brumm MC, et al. Implications of starvationinduced change in right dorsal anterior cingulate volume in anorexia nervosa. Int J Eat Disord. 2008;41(7):602-610.

42. Bailer UF, Frank GK, Henry SE, et al. Altered brain serotonin 5-HT1A receptor binding after recovery from anorexia nervosa measured by positron emission tomography and [carbonyl11C] WAY-100635. Arch Gen Psychiatry. 2005;62(9):1032-1041.

43. Uher R, Brammer MJ, Murphy T, et al. Recovery and chronicity in anorexia nervosa: brain activity associated with differential outcomes. Biol Psychiatry. 2003;54(9):934-942.

44. Keating $C$. Theoretical perspective on anorexia nervosa: the conflict of reward. Neurosci Biobehav Rev. 2010;34(1):73-79.

45. Keating C, Tilbrook AJ, Rossell SL, Enticott PG, Fitzgerald PB. Reward processing in anorexia nervosa. Neuropsychologia. 2012;50(5): 567-575.

46. Holliday J, Tchanturia K, Landau S, Collier D, Treasure J. Is impaired set-shifting an endophenotype of anorexia nervosa? Am J Psychiatry. 2005;162(12):2269-2275.
Neuropsychiatric Disease and Treatment

\section{Publish your work in this journal}

Neuropsychiatric Disease and Treatment is an international, peerreviewed journal of clinical therapeutics and pharmacology focusing on concise rapid reporting of clinical or pre-clinical studies on a range of neuropsychiatric and neurological disorders. This journal is indexed on PubMed Central, the 'PsycINFO' database and CAS,

\section{Dovepress}

and is the official journal of The International Neuropsychiatric Association (INA). The manuscript management system is completely online and includes a very quick and fair peer-review system, which is all easy to use. Visit http://www.dovepress.com/testimonials.php to read real quotes from published authors. 